\title{
Нормативная система системно-объектного анализа и моделирования
}

\author{
В.В. Михелёв ${ }^{1}$, С.И. Маторин ${ }^{2}$, А.Г. Жихарев ${ }^{1}$ \\ ${ }^{1)}$ Белгородский государственный национальный исследовательский университет, \\ Россия, г. Белгород, ул. Победы, 85 \\ 2) ЗАО «СофтКоннект», Россия, 308023, г. Белгород, ул. Студенческая, 19, кор. 1 \\ E-mail: keeper121@ya.ru, matorin@ softconnect.ru, zhikharev@bsu.edu.ru
}

\begin{abstract}
Аннотация
В статье обсуждается возможность создания формально-семантической нормативной системы системно-объектного анализа и моделирования. Система рассматривается в рамках системнообъектного подхода в виде конструкции «Узел-Функция-Объект», формализуемой с помощью дескрипционной логики. Предложена классификация систем, которая использует классификацию потоков связей и узлов, входящую в концепцию системно-объектного подхода. Представлена концептуальная классификационная схема для системных компонент. Описаны её свойства, на ее основе разрабатывается способ задания формального алфавита узлов связей систем, имеющего конкретное предметное содержание. Также представлены правила манипулирования получаемыми алфавитными символами. Обосновывается возможность и целесообразность построения нормативной системы, обладающей повышенными выразительными возможностями. Рассматривается пример использования формально-семантического алфавита системных элементов, демонстрирующий возможности упрощения процедуры графоаналитического моделирования сложной системы.
\end{abstract}

Ключевые слова: системно-объектный подход, формализация, элемент «Узел-Функция-Объект», дескрипционная логика ALCHOIQ(D), нормативная система, алфавитные узлы.

Благодарности: работа поддержана грантами РФФИ 18-07-00355a, 18-07-00356a, 19-07-00290а и № 19-07-00111 a, 19-29-01047мк.

Для цитирования: Михелёв В.В., Маторин С.И., Жихарев А.Г. 2020. Нормативная система системнообъектного анализа и моделирования. Экономика. Информатика. 47 (3): 623-637. DOI 10.18413/26870932-2020-47-3-623-637.

\section{Normative system of system-object analysis and modeling}

\author{
V.V. Mikhelev', S.I. Matorin' ${ }^{2}$, A.G. Zhikharev ${ }^{1}$ \\ 1) Belgorod State National Research University, \\ 85 Pobedy St., Belgorod, 308015, Russia \\ 2) «SoftConnect» CJSC, 19 cor.1 Student St., Belgorod, 308023, Russia \\ E-mail: keeper121@ya.ru,matorin@softconnect.ru, zhikharev@bsu.edu.ru
}

\begin{abstract}
The article discusses the possibility of creating a formal-semantic normative system of system-object analysis and modeling. The system is considered in the framework of the system-object approach in the form of a design "Union-Function-Object", formalized using descriptive logic. A classification of systems is proposed that uses the classification of communication flows and nodes, which is part of the concept of a system-object approach. A conceptual classification scheme for system components is presented. Its properties are described; on its basis, a method is being developed for specifying the formal alphabet of system connection nodes with a specific objective content. Also presented are the rules for manipulating the resulting alphabetical characters. The possibility and expediency of building a regulatory system with enhanced expressive capabilities are substantiated. An example of the use of the formal semantic alphabet of system elements is considered, which demonstrates the possibility of simplifying the procedure of graphic-analytical modeling of a complex system.
\end{abstract}


Keywords: system-object approach, formalization, element «Union-Function-Object», ALCHOIQ(D) descriptive logic, normative system, alphabet nodes.

Acknowledgements: the research is financially supported by the projects of the Russian Foundation for Fundamental Research No 18-07-00355a, 18-07-00356a, 19-07-00290a and № 19-07-00111a, 19-29$01047 \mathrm{mk}$.

For citation: Mikhelev V.V., Matorin S.I., Zhikharev A.G. 2020. Normative system of system-object analysis and modeling. Economics. Information technologies. 47 (3): 623-637 (in Russian). DOI: 10.18413/26870932-2020-47-3-623-637.

\section{Введение}

Системный анализ представляет собой так называемую «нормативную методологию» [Никаноров, 1969]. В развитии этой идеи в работах [Кондаков, 1975; Горский, 1990] вводится понятие нормативная система для описания метода системного анализа. Нормативная система представляет построенную генетическим, а не аксиоматическим способом формальную систему. Такая система должна использовать некоторый алфавит и синтаксис, как правила манипулирования им, причём без использования исходных постулируемых утверждений (аксиом). «Генетический способ характеризуется прежде всего тем, что в качестве исходных он рассматривает некоторую совокупность объектов, из которых по определенным правилам строятся затем новые объекты» [Горский, 1990, с. 54]. Примерами формальных систем, построенных генетическим способом, являются теория паттернов Гренандера, исчисление процессов Милнера и теория объектов Абади-Кардели.

Частью любой нормативной системы (как и любой формальной системы/теории) является алфавит. Он включает в себя знаки (символы), используемые для записи по определенным правилам выражений (формул) и осуществления вывода. Считается, что эти знаки рассматриваются формально, без какой бы то ни было содержательной интерпретации. «Интерпретацию знаки формальной системы могут получить (а могут и не получить) уже после создания теории» [Петров, 1977; Маслов, 1986].

«Наука о знаковых системах бескомпромиссно утверждает, что любой знак всегда представляет собой единство означающего и означаемого, формы и содержания» [Степанова, 1983]. Таким образом, знаки алфавита нормативной (формальной) системы, должны иметь содержательную интерпретацию до включения их в какое-либо выражение. При этом применение знаков любой формальной системы всегда предполагает знание и использование их смысла. Например, совершенно очевидно, что выражения «A $\wedge$ B» и «A $\vee \mathrm{B} »$ различаются между собой не только по форме значка, стоящего между «А» и «В», но, в первую очередь, по стоящему за этой формой смыслу.

Гильбертовское заблуждение (получившее, к сожалению, широкое распространение [Гильберт, 1979]) относительно отсутствия содержания у знаков нормативной (формальной) системы и является источником причины, по которой традиционная математика не в состоянии описывать весьма содержательные собственно системные свойства и отношения.

Естественный язык является знаковой системой и позволяет понять, как предметноориентированное содержание может быть приписано знакам алфавита формальной системы. Например, в языке делового общения, содержащем термины, имеющие понятийное содержание, «исходная совокупность слов составляет иерархическую систему понятий (терминов), т. е. концептуальную классификационную структуру» [Matorin, 1998, 1 и 2]. Такая структура обладает свойством адаптивности к предметной области, так как слова могут и добавляться, и удаляться из нее. Следовательно, есть возможность использовать концептуальную классификационную схему для определения смысла алфавитных символов нормативной системы. С ее помощью может быть решена задача построения нормативной системы, обладающей повышенными выразительными возможностями. 
Рассмотрим каким образом может быть построена нормативная система системнообъектного анализа с помощью концептуальной классификационной модели и какими особенностями она будет обладать.

\section{1. Особенности системно-объектного подхода и его формализация средствами дескрипционной логики}

В системно-объектном подходе система рассматривается как «функциональный объект, функция которого обусловлена функцией объекта более высокого яруса» (т. е. функцией надсистемы), что в целом соответствует пониманию системы, данному в работе [Мельников, 1978]. «Подобное содержательное определение системы уточнено авторами путем представления системы в виде триединой конструкции «Узел-Функция-Объект» (УФО-элемент), в которой узел-перекресток входных и выходных связей/потоков системы, функция - процесс преобразования входных потоков в выходные, объект - совокупность субстанциальных характеристик системы» [Теория систем ..., 2020]. Такое представление системы позволяет, в свою очередь, используя исчисление объектов Абади-Кардели [Abadi, Cardelli, 1996], считать систему s = [us; fs; Os] специальным объектом данного исчисления, состоящим из полей и методов, который будем называть узловым объектом [Жихарев и др., 2015].

«Здесь узел системы us $\leftrightarrow$ Ls? $\cup$ Ls! формально представляет собой поля узлового объекта для описания объектов еще одного специального вида, называемых нами потоковыми объектами [Жихарев и др., 2015], которые соответствуют множеству функциональных связей данной системы; Ls? - множество входящих интерфейсных потоковых объектов, которые соответствуют входящим связям системы s; Ls! - множество исходящих интерфейсных потоковых объектов, которые соответствуют выходящим связям системы $\mathbf{s} ; \mathbf{L s} ? \subset \mathbf{L}$ и $\mathbf{L s} ! \subset \mathbf{L}$, т. е. относятся к множеству всех связей $\mathbf{L}$ (потоковых объектов)».

«Функция системы fs: Ls? $\rightarrow$ Ls! формально представляет собою метод узлового объекта, т. е. процесс преобразования входящих интерфейсных потоковых объектов (входящих связей системы) Ls? в исходящие интерфейсные потоковые объекты (выходящие связи системы) Ls!».

«Объект системы Os = Os? $\cup$ Os! $\cup$ Osf формально представляет собой множество полей узлового объекта для описания объектных (субстанциальных) характеристик системы. Множество полей для описания объектных характеристик системы состоит из трех подмножеств: Os? - множество полей, которое содержит интерфейсные входные характеристики узлового объекта (т. е. показатели входных связей системы), Os! - множество полей, которое содержит интерфейсные выходные характеристики узлового объекта (т. е. показатели выходных связей системы s), Osf - множество полей, которое содержит передаточные характеристики узлового объекта (показатели внутренних связей системы)».

В рамках системно-объектного подхода рассматриваются не абстрактные связи/отношения $\mathbf{L}$, а связи/отношения, имеющие определенное предметное содержание. Это обусловлено введением в концепцию системно-объектного подхода классификации связей. В данной классификации абстрактный класс «Связь (L)» делится на непересекающиеся подклассы «Материальная связь (m)» и «Информационная связь (i)»; класс материальных связей делится на непересекающиеся подклассы «Вещественная связь (v)» и «Энергетическая связь (e)»; класс информационных связей - на непересекающиеся подклассы «Связь по данным (d)» и «Управляющая связь (c)» [Теория систем ..., 2020]. Данное обстоятельство и позволяет создать нормативную систему системно-объектного анализа с помощью концептуальной классификационной модели, определяющей алфавит связей.

Для формального описания упомянутых выше элементов системно-объектного подхода можно использовать выразительные возможности дескрипционной логики. Дескрипционная логика (ДЛ) является логическим языком описания понятий и связей между ними с помощью индивидов, концептов и ролей [Baader et al., 2003]. Для наших целей можно ввести логику ALCHOIQ(D) на основе базовой логики ALC, такая логика обладает следующим синтаксисом (записанным в краткой форме): 


$$
\left\{\mathrm{T} ; \perp ; \mathbf{A} ; \mathbf{A} \subseteq \mathbf{C} ; \neg \mathbf{C} ; \mathbf{C} \sqcap \mathbf{B} ; \mathbf{C} \sqcup \mathbf{B} ; \exists \mathbf{R} . \mathbf{C} ; \forall \mathbf{R} . \mathbf{C} ; \geq \mathbf{n} \mathbf{R} . \mathbf{C} ;\{\mathbf{a}\} ; \exists\left[\mathbf{u}_{1}, \ldots, \mathbf{u}_{\mathbf{n}}\right] . \mathbf{P}\right\},
$$

где $\mathbf{A}$ и $\mathbf{C}, \mathbf{B}$ - атомарные концепты, $\mathbf{R}$ - атомарная роль, Т и $\perp$ - концепты, представляющие истину и ложь; $\{\mathbf{a}\}$ - описание индивида с помощью понятия «номинал» [Baader et al., 2003].

Семантика любой дескрипционной логики задается с помощью понятия интерпретации [Schmidt-Schauss, Smolka, 1991]. Интерпретация есть пара $\mathrm{I}=\left(\Delta,{ }^{\prime}\right)$, состоящая из непустого множества $\Delta$, называемого областью данной интерпретации, и интерпретирующей функции .I , которая сопоставляет:

- каждому атомарному концепту $\mathbf{A} \in \mathbf{C N}$ - произвольное множество $\mathbf{A}^{\mathbf{I}} \subseteq \Delta$;

- каждой атомарной роли $\mathbf{R} \in \mathbf{R} \mathbf{N}-$ произвольное множество $\mathbf{R}^{\mathbf{I}} \subseteq \Delta \times \Delta$.

$\mathbf{C N}=\left\{\mathbf{A}_{\mathbf{1}}, \ldots, \mathbf{A}_{\mathbf{m}}\right\}$ и $\mathbf{R} \mathbf{N}=\left\{\mathbf{R}_{\mathbf{1}}, \ldots, \mathbf{R}_{\mathbf{n}}\right\}-$ конечные непустые множества атомарных концептов и атомарных ролей.

Для концептов логики существует несколько базовых правил:

- $\mathrm{T}^{\mathrm{I}}=\Delta, \perp^{\mathrm{I}}=\emptyset,(\neg \boldsymbol{C})^{\boldsymbol{I}}=\Delta \backslash \boldsymbol{C}^{\boldsymbol{I}}$;

- $(\mathbf{C} \sqcap \mathbf{D})^{\mathrm{I}}=\mathbf{C}^{\mathrm{I}} \cap \mathbf{D}^{\mathrm{I}}$;

- $(\mathbf{C} \sqcup \mathbf{D})^{\mathrm{I}}=\mathbf{C}^{\mathrm{I}} \cup \mathbf{D}^{\mathrm{I}}$;

- $(\exists \mathbf{R} . \mathbf{C})^{\mathbf{I}}=\left\{\mathbf{e} \in \Delta \mid\right.$ существует $\mathbf{d} \in \Delta$ такой, что $\langle\mathbf{e}, \mathbf{d}\rangle \in \mathbf{R}^{\mathbf{I}}$ и $\left.\mathbf{d} \in \mathbf{C}^{\mathbf{I}}\right\}$;

- $(\forall \mathbf{R} . \mathbf{C})^{\mathbf{I}}=\left\{\mathbf{e} \in \Delta \mid\right.$ для всех $\mathbf{d} \in \Delta$ таких, что $\langle\mathbf{e}, \mathbf{d}\rangle \in \mathbf{R}^{\mathbf{I}}$, выполнено $\left.\mathbf{d} \in \mathbf{C}^{\mathbf{I}}\right\}$.

Например, пусть областью интерпретации I будет множество $\Delta$ всех биологических особей. Атомарные концепты: Animal, Cat, Human, Male - множества всех животных, кошек, людей, особей мужского пола. Концепты обладают атомарными ролями hasParent и hasChild, показывающими связь концептов. Таким образом, пара $\langle e, d\rangle$ принадлежит отношению hasChild, если $d$ является ребенком $e$, а отношение hasParent ${ }^{I}$ является обратным к hasChild . При такой интерпретации составные концепты обретают следующий смысл. Male п Cat множество котов (мужские особи) или Male $\sqcap$ ヨhasParent. (Human $\square \neg$ Male) - множество особей мужского пола, у которых есть родитель, являющийся человеком не мужского пола.

Роли используются для описания двуместных отношений (в базовой ДЛ). Например, «X есть родитель для Y». X и Y - концепты, представляющие произвольные объекты или классы. Концепты используются для описания классов некоторых объектов, например, «Люди», «Животные», «Машины». Индивид представляет собой конкретное понятие предметной области, экземпляр класса, например, «Мария», «Иван», «Москва».

В дополнение, $\geq \mathbf{n ~ R . C ~ - ~ о п и с ы в а е т ~ к о л и ч е с т в е н н о е ~ о г р а н и ч е н и е ~ н а ~ р о л ь ~ к о н ц е п т а ~}$ [Baader, Sattler, 1999]; $\mathbf{u}_{1}, \ldots, \mathbf{u}_{\mathbf{n}}-$ произвольные конкретные атрибуты; $\mathbf{P}-n$-местный конкретный предикат, где $\mathbf{P} \in \mathbf{P N}$ и $\mathbf{P N}$ - множество предикатных символов определённых на «конкретной области» D [Tessaris, 2001].

Конкретная область (D) - это пара $\mathbf{D}=(\mathbf{D}, \mathbf{\Phi})$, где $\mathbf{D}-$ непустое множество, a $\boldsymbol{\Phi}$ - набор предикатов на множестве D. Каждый символ $\mathbf{P} \in \mathbf{P N}$ имеет валентность $n$, а $\boldsymbol{\Phi}$ сопоставляет ему $n$-местное отношение $\mathbf{P}^{\mathbf{D}} \subseteq \mathbf{D}^{\mathbf{n}}$.

Обычно, конкретная область $\operatorname{Order}_{\mathbf{N}}=(\mathbf{N}, \boldsymbol{\Phi})$ представляет собой множество натуральных чисел $\mathbf{N}$ и семейство предикатов $\boldsymbol{\Phi}$. $\boldsymbol{\Phi}$ состоит из двуместных предикатов $<, \leq,=$ (и их отрицания: $\neq, \geq,>$ ) и одноместных предикатов $<_{n}, \leq_{n},=_{n}$ (и их отрицания: $\neq_{n},>_{n},>_{n}$ ). Пусть hasAge есть конкретный атрибут (со значениями в D), обозначающий возраст человека. Тогда следующий концепт задает множество совершеннолетних людей, то есть возраст которых не менее 18 лет: Human $\sqcap$ ヨhasAge. $\geq_{18}$.

Классические ДЛ позволяют описывать общие понятия о концептах и их взаимосвязях с помощью набора терминологических аксиом - ТВох. Существует возможность описания знаний об индивидуальных объектах, их связях и свойствах в терминах ДЛ - АВох. Также в ДЛ ALCHIO(D) введено понятие иерархии ролей, которую также можно представить в виде набора аксиом - RBox. 2019]:

B терминах ДЛ можно описать концепты, составляющие УФО-элементы [Михелев,

Узел, как перекресток множества входов Ls? и выходов Ls!: 


$$
\mathbf{U}=\mathbf{L s} ? \sqcup \mathbf{L s} \text { ! }
$$

Функция, как преобразование множества входов в множество выходов:

$$
\mathbf{F}=\mathbf{L s} ! \Pi \exists \mathbf{R} . \mathbf{L s} \text {. }
$$

Выражение Ls! $П \exists \mathbf{R}$. Ls? обозначает множество выходов Ls!, «связанных» ролью R с множеством входов Ls?; R - функциональная роль, показывающая соответствие между концептами. Можно ввести роль $\mathbf{R}$ - «имеетСоответствие», тогда можно уточнить определение функции:

$$
\mathbf{F}=\mathbf{L s} ! \sqcap \exists \text { имеетСоответствие. } \mathbf{L s} \text {. }
$$

Объект: реализует функцию и обладает интерфейсными и субстанциональными характеристиками:

$$
\mathbf{O}=\mathbf{O S} \text { ? } \sqcup \text { OS! ப OSf. }
$$

Система $\mathbf{s}$, в терминах ДЛ представляется как совокупность приведённых выше выражений для составляющих УФО-элемента:

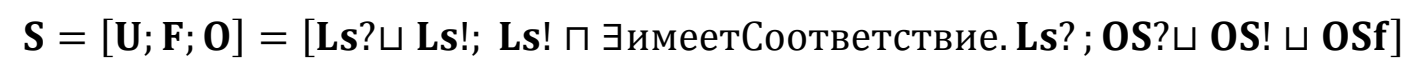

C помощью ДЛ ALCHIO(D) легко описать упомянутую выше классификацию связей/отношений в виде ТВох:

Иерархия концептов:

Материальная связь (m) ᄃ Связь (L);

Информационная связь (i) ᄃ Связь (L);

Вещественная связь (v) ᄃ Материальная связь (m);

Энергетическая связь (e) ᄃ Материальная связь $(\mathbf{m})$;

Связь по данным (d) ᄃ Информационная связь (i);

Управляющая связь (c) ᄃ Информационная связь (i);

Правила построения конщептов (связей):

Связь (L) $\equiv$ Материальная связь (m) $\sqcup$ Информационная связь (i);

Материальная связь (m) $\equiv$ Вещественная связь (v) ப Энергетическая связь (e);

Информационная связь (i) $\equiv$ Связь по данным (d) ப Управляющая связь (c);

Выше представленный набор терминологических аксиом представляет собой классификацию связей по типу сущности на непересекающиеся классы.

\section{2. Алфавит нормативной системы системно-объектного анализа}

При использовании для создания алфавита нормативной системы классификационной модели алфавитный набор символов представляет собой совокупность конкретных классов (листьев) классификационной схемы, а также совокупность соответствующих экземпляров этих классов. Заданный таким образом алфавит состоит из символов, однозначно интерпретируемых по своим дефинициям (свойствам) и пользователем, и компьютером за счет фиксации в структуре классификации родо-видовых определений (дефиниций), т. е. ее параметричности, что позволяет рассматривать данный алфавит как формально-семантический (и, соответственно, формальносемантической считать саму нормативную систему).

Для превращения предложенной иерархии классов связей/отношений в алфавитный набор узлов зададим правила манипулирования символами алфавита связей. Этими правилами в данном случае будут правила создания элементарных (алфавитных) узлов как перекрестков связей, т. е. структурных элементов, способных вступать в определенные отношения, задаваемые с помощью фасетной классификации.

Правила создания узлов на уровне вещественных и энергетических потоков на уровне потоков данных и потоков управления и на уровне смешанных потоков (см. таблицу 1) обусловлены тем, что вещество не может преобразовываться в энергию в чистом виде, данные в управление, а энергия не может преобразоваться в вещество, управление в данные (классы вещественных и энергетических потоков, а также данных и управления не пересекаются). 
Алфавитные узлы

Alphabetic nodes

\begin{tabular}{|l|l|l|l|l|l|l|l|l|l|l|}
\hline & $\mathbf{v}$ ! & e! & v?, e? & d! & c! & d?, c? & v!, d! & v!, c! & e!, d! & e!, c! \\
\hline v? & $*$ & & & & & & & & & \\
\hline e? & & $*$ & & & & & & & & \\
\hline v?, e? & & & $*$ & & & & & & & \\
\hline d? & & & & $*$ & & & & & & \\
\hline c? & & & & & $*$ & & & & & \\
\hline d?, c? & & & & & & $*$ & & & & \\
\hline v?, d? & & & & & & & $*$ & & & \\
\hline v?, c? & & & & & & & & $*$ & & \\
\hline e?, d? & & & & & & & & & $*$ & \\
\hline e?, c? & & & & & & & & & & $*$ \\
\hline
\end{tabular}

Представленная фасетная классификация узлов (табл. 1) соответствует родовидовой (таксономической) классификации компонент системы (рис. 1), объединяющей классификацию связей и элементов (функциональных узлов), которая является развитием классификации компонент и свойств системы, описанной в работе [Маторин и др., 2016]. Данная родовидовая классификация позволяет сформулировать определения (родовидовые) алфавитным связям и алфавитным функциональным узлам (см. табл. 2 и 3 ).

Таблица 2

Table 2

Алфавитный набор связей / отношений

Alphabetical set of links / relationships

\begin{tabular}{|c|c|c|c|}
\hline ЗНАК & ТЕРМИН & $\begin{array}{l}\text { РОДОВОЕ } \\
\text { ПОНЯТИЯ }\end{array}$ & ВИДОВОЕ ОТЛИЧИЕ \\
\hline $\mathbf{v}$ & $\begin{array}{l}\text { Вещественная } \\
\text { связь }\end{array}$ & $\begin{array}{l}\text { Материальная } \\
\text { связь (m) }\end{array}$ & $\begin{array}{l}\text { Создающая вещественную структуру системы, } \\
\text { определяющуюся отношением порядка на } \\
\text { множестве V - vRv }\end{array}$ \\
\hline $\mathbf{e}$ & $\begin{array}{l}\text { Энергетическая } \\
\text { связь }\end{array}$ & $\begin{array}{l}\text { Материальная } \\
\text { связь }(\mathbf{m})\end{array}$ & \begin{tabular}{l}
\multicolumn{3}{l}{ Создающая энергетическую структуру системы, } \\
определяющуюся отношением порядка на \\
множестве E-eRe
\end{tabular} \\
\hline d & Связь по данным & $\begin{array}{l}\text { Информационная } \\
\text { связь (i) }\end{array}$ & $\begin{array}{l}\text { Создающая структуру данных системы, } \\
\text { определяющуюся отношением порядка на } \\
\text { множестве D - dRd }\end{array}$ \\
\hline c & $\begin{array}{l}\text { Связь по } \\
\text { управлению }\end{array}$ & $\begin{array}{l}\text { Информационная } \\
\text { связь (i) }\end{array}$ & $\begin{array}{l}\text { Создающая структуру управления системы, } \\
\text { определяющуюся отношением порядка на } \\
\text { множестве } \mathrm{C}-\mathrm{cRc}\end{array}$ \\
\hline
\end{tabular}

Алфавитный набор узлов

Alphabetical set of nodes

\begin{tabular}{|c|c|c|}
\hline ЗНАК & $\begin{array}{l}\text { РОДОВОЕ } \\
\text { ПОНЯТИЕ }\end{array}$ & ВИДОВОЕ ОТЛИЧИЕ \\
\hline $\mathbf{V}$ & $\mathrm{M}$ & Определяющийся преобразованием $\boldsymbol{V} \boldsymbol{v}$ \\
\hline $\mathbf{E}$ & M & Определяющийся преобразованием $\boldsymbol{E} \boldsymbol{e}$ \\
\hline VE & $\mathrm{M}$ & Определяющийся преобразованием $\boldsymbol{V E \boldsymbol { E }}$ \\
\hline D & I & Определяющийся преобразованием $\boldsymbol{D} \boldsymbol{d}$ \\
\hline $\mathbf{C}$ & I & Определяющийся преобразованием $\boldsymbol{C c}$ \\
\hline
\end{tabular}




\begin{tabular}{|c|c|c|}
\hline ЗНАК & $\begin{array}{l}\text { РОДОВОЕ } \\
\text { ПОНЯТИЕ }\end{array}$ & ВИДОВОЕ ОТЛИЧИЕ \\
\hline DC & $\mathrm{I}$ & Определяющийся преобразованием $\boldsymbol{D C d}$ \\
\hline VD & MI & Определяющийся преобразованием VDvd \\
\hline VC & MI & Определяющийся преобразованием $\boldsymbol{V C v \boldsymbol { c }}$ \\
\hline ED & MI & Определяющийся преобразованием EDed \\
\hline EC & MI & Определяющийся преобразованием $\boldsymbol{E C e c}$ \\
\hline
\end{tabular}

Полученный алфавит узловых элементов можно формально описать средствами упомянутой выше ДЛ в виде ТВох следующим образом:

Иерархия кониептов:

$\mathbf{V} \sqsubset \mathbf{M} ; \mathbf{E} \sqsubset \mathbf{M} ; \mathbf{V E} \sqsubset \mathbf{M}$

$\mathrm{D} \sqsubset \mathrm{I} ; \mathrm{C} \sqsubset \mathrm{I} ; \mathrm{DC} \sqsubset \mathrm{I}$;

VD $\sqsubset \mathrm{MI} ; \mathrm{VC} \sqsubset \mathrm{MI} ; \mathrm{ED} \sqsubset \mathrm{MI} ; \mathrm{EC} \sqsubset \mathrm{MI}$.

Правила построения концептов (алфавитный набор узлов):

$\mathbf{V} \equiv \mathbf{v} ! \sqcap \exists$ имеетСоответствие. (v?);

$\mathbf{E} \equiv \mathbf{e} !$ П ЭимеетСоответствие. (e?);

$\mathbf{D} \equiv \mathbf{d} !$ П ЭимеетСоответствие. (d?);

$\mathbf{C} \equiv \mathbf{c} ! \sqcap \exists$ имеетСоответствие. (c) $)$

$\mathbf{V E} \equiv(\mathbf{v} ! \sqcup \mathbf{e} !) \sqcap$ ヨимеетСоответствие. (v?ப e? );

$\mathbf{V D} \equiv(\mathbf{v} ! \sqcup \mathbf{d} !) \sqcap$ ヨимеетСоответствие. (v? $\sqcup \mathbf{d} ?)$;

$\mathbf{V C} \equiv(\mathbf{v} ! \sqcup \mathbf{c} !) \sqcap \exists$ ИмеетСоответствие. $(\mathbf{v} ? \sqcup \mathbf{c} ?) ;$

$\mathbf{E D} \equiv(\mathbf{e} ! \sqcup \mathbf{d} !) \sqcap$ ヨимеетСоответствие. $(\mathbf{e} ? \sqcup \mathbf{d} ?)$;

$\mathbf{E C} \equiv(\mathbf{e} ! \sqcup \mathbf{c} !) \sqcap \exists$ имеетСоответствие. (e?ப $\mathbf{c}$ ? $)$;

DC $\equiv(\mathbf{d} ! \sqcup \mathbf{c} !) \sqcap$ ヨимеетСоответствие. $(\mathbf{d}$ ? $\sqcup \mathbf{c} ?)$.

\section{3. Правила использования символов введенного алфавита}

Использование содержательной классификации связей / отношений позволяет рассматривать не бесчисленное множество видов структурных элементов систем (т. е. Узлов), а весьма ограниченный их набор. При этом правила оперирования этими элементами (т. е. алфавитными символами нормативной системы) должны быть основаны на системных отношениях, рассматриваемых в рамках выбранного системного подхода.

Как известно, системно-объектный подход рассматривает в качестве основного системного отношения вслед за автором работы [Мельников, 1978] «отношение поддержания функциональной способности целого».

Соблюдение данного отношения обеспечивается путем выполнения следующих «правил системной композиции», предложенных в работе [Маторин, 2005] и уточненных нами в рамках данного исследования впервые с помощью дескрипционной логики:

1. «Присоединения»: элементы могут быть связаны только в том случае, если выходные и входные потоки одноименные (из классификации связей).

Пусть $\boldsymbol{S}_{\mathbf{i}}$ и $\mathbf{S}_{\mathbf{j}}$ - системы, как элементы «Узел-Функция-Объект», описываемые с помощью ДЛ в виде выражения (2). Тогда данное правило можно описать как показано ниже.

$\exists \boldsymbol{S}: \mathbf{S} \equiv \boldsymbol{S}_{\mathbf{i}} \sqcup \boldsymbol{S}_{\mathbf{j}}$, если системы $\boldsymbol{S}_{\mathbf{i}}$ и $\boldsymbol{S}_{\mathbf{j}}$ рассматриваются как узлы, принадлежащие к одному классу (типу) узлов Ls: $\mathbf{L} \boldsymbol{s} !_{\mathbf{i}}=\mathbf{L} \boldsymbol{s} ?_{\mathbf{j}}$, т. е. ЭимеетПредка. $\boldsymbol{S}_{\mathbf{i}}=$ ЭимеетПредка. $\boldsymbol{S}_{\mathbf{j}}$.

2. «Баланса»: присоединенные по первому правилу элементы образуют систему, если выходные потоки первого элемента могут быть полностью поглощены как входные потоки присоединяемого элемента.

В соответствии с правилом присоединения: $\mathbf{L} \boldsymbol{s} !_{\mathbf{i}}=\mathbf{L} \boldsymbol{s} ?_{\mathbf{j}}$. Т. е. входному потоку $\mathbf{L} \boldsymbol{s} ?_{\mathbf{j}}$, как аргументу функции, соответствует выходной функциональный поток $\mathbf{L s} !_{\mathbf{i}}$. Таким образом, для выполнения данного правила область значения функции $\mathbf{F}_{\boldsymbol{i}}$ должна входить в область определения функции $\mathbf{F}_{\mathbf{j}}: \operatorname{ran} \mathbf{F}_{i} \subseteq \operatorname{dom} \mathbf{F}_{\mathbf{j}}$. 


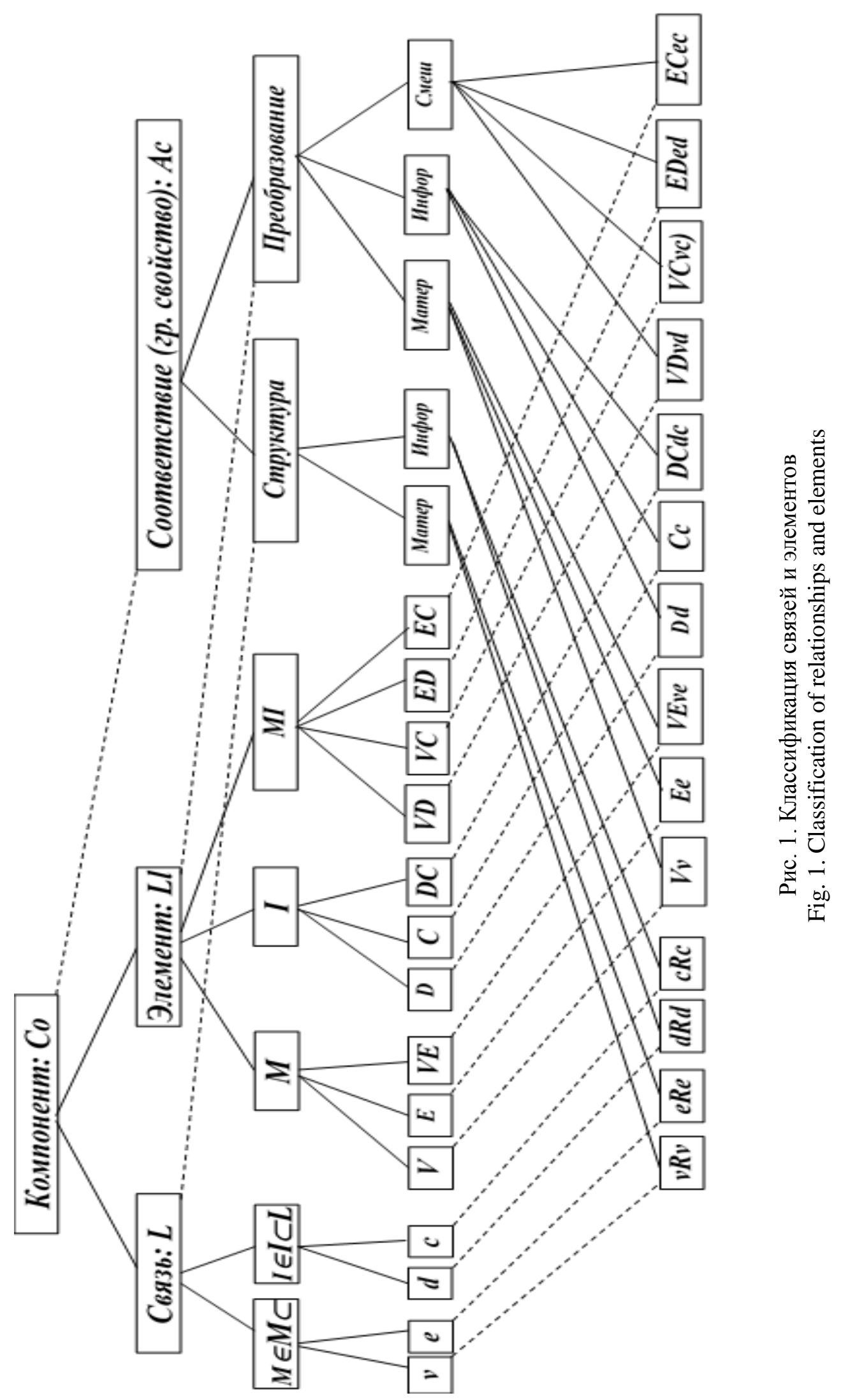


3. «Реализации»: присоединенные по первому правилу элементы образуют систему, если их интерфейсы соответствуют друг другу.

В соответствии с приведёнными ранее правилами. $\exists \mathbf{S : S} \equiv \mathbf{S}_{\mathbf{i}} \sqcup \mathbf{S}_{\mathbf{j}}$, если $\mathbf{O S} !_{\mathbf{S}_{\mathbf{i}}} \leftrightarrow \mathbf{O S} \mathbf{s}_{\mathbf{j}}$, т. е. если обеспечено соответствие интерфейсов систем.

4. «Замкнутости»: внутренний поток, не связанный так или иначе с «проточными» потоками от входа системы к ее выходу замкнут, т. е. образует цикл.

Пусть $\mathbf{S} \equiv[\mathbf{L s}$ ? ப Ls! ; Ls! П $\exists \mathbf{R}$. Ls? ; OS? $\mathbf{~ O S ! ~ ப ~ O S f ] , ~ т о г д а ~ в н у т р е н н и е ~ п о т о к и , ~ н е ~}$ связанные с Ls? и Ls!, образуемые между подсистемами $\left(\exists \boldsymbol{S}_{\mathbf{i}} \subset \mathbf{S}\right)$, замкнуты, исходя из правила баланса для этих систем.

Следующим логичным шагом описания правил использования алфавита является описание операций над системами в соответствии с отмеченными выше правилами. При этом ДЛ позволяет описать эти операции, развивая результаты, представленные в работе [Михелев, 2019].

Пусть даны две системы $\boldsymbol{S}_{\mathbf{i}}$ и $\boldsymbol{S}_{\mathbf{j}}$, описываемые в соответствии с выражением (2):

$$
\begin{aligned}
& \mathbf{S}_{\mathbf{i}}=\left[\mathbf{L s} ?_{\mathbf{i}} \sqcup \mathbf{L s} !_{\mathbf{i}} ; \mathbf{L s} !_{\mathbf{i}} \sqcap \text { ヨимеетСоответствие. Ls? } ?_{\mathbf{i}} ; \mathbf{O S} ?_{\mathbf{i}} \sqcup \mathbf{O S} !_{\mathbf{i}} \sqcup \mathbf{O S f}_{\mathbf{i}}\right] \\
& \mathbf{S}_{\mathbf{j}}=\left[\mathbf{L s} ?_{\mathbf{j}} \sqcup \mathbf{L s} !_{\mathbf{j}} ; \mathbf{L s} !_{\mathbf{j}} \sqcap \text { ヨимеетСоответствие. } \mathbf{L} \boldsymbol{s} ?_{\mathbf{j}} ; \mathbf{O S} ?_{\mathbf{j}} \sqcup \mathbf{O S} !_{\mathbf{j}} \sqcup \mathbf{O S f}_{\mathbf{j}}\right]
\end{aligned}
$$

Представим ниже ряд базовых операций.

\section{Операция соединения систем.}

Системы $\mathbf{S}_{\mathbf{i}}$ и $\mathbf{S}_{\mathbf{j}}$ можно соединить при соблюдении следующих условий: $\mathbf{L} \boldsymbol{s} !_{\mathbf{i}}=\mathbf{L s} ?_{\mathbf{j}}$, $\boldsymbol{r a n} \mathbf{F}_{\mathbf{i}} \subseteq \boldsymbol{d o m} \mathbf{F}_{\mathbf{j}}, \quad \mathbf{O S} !_{\mathbf{i}} \leftrightarrow \mathbf{O S} ?_{\mathbf{j}}$. Данный результат формально можно получить путем объединения потоков/связей системы $\boldsymbol{S}_{\mathbf{i}}$ и потоков/связей системы $\boldsymbol{S}_{\mathbf{j}} \mathbf{c}$ последующим вычитанием из этого объединения результата пересечения потоков/связей $\mathbf{S}_{\mathbf{i}}$ и $\mathbf{S}_{\mathbf{j}}$. Это позволит удалить из рассмотрения на уровне нового соединенного узлового объекта (системы $\mathbf{S}_{\mathrm{ij}}$ ) внутренние связи, за счет которых и происходит присоединение $\boldsymbol{S}_{\boldsymbol{i}}$ к входу $\mathbf{S}_{\mathbf{j}}$. Таким образом, объединяя узлы, получим: $\mathbf{U}_{\mathbf{i j}}=\left(\mathbf{L s} ?_{\mathbf{i}} \sqcup \mathbf{L s} !_{\mathbf{i}}\right) \sqcup\left(\mathbf{L s} ?_{\mathbf{j}} \sqcup \mathbf{L s} !_{\mathbf{j}}\right)-\left(\mathbf{L s} ?_{\mathbf{i}} \sqcup \mathbf{L s} !_{\mathbf{i}}\right) \sqcap\left(\mathbf{L s} ?_{\mathbf{j}} \sqcup \mathbf{L s} !_{\mathbf{j}}\right)=$ $=\mathbf{L s} ?_{\mathbf{i}} \sqcup \mathbf{L s} !_{\mathbf{j}} \sqcup \mathbf{L s} ?_{\mathbf{j}} \sqcup \mathbf{L s} !_{\mathbf{j}}-\mathbf{L s} ?_{\mathbf{i}} \sqcap \mathbf{L s} ?_{\mathbf{j}} \sqcup \mathbf{L s} !_{\mathbf{i}} \sqcap \mathbf{L s} ?_{\mathbf{j}} \sqcup \mathbf{L s} ?_{\mathbf{i}} \sqcap \mathbf{L s} !_{\mathbf{j}} \sqcup \mathbf{L s} ?_{\mathbf{i}} \sqcap \mathbf{L s} !_{\mathbf{j}}$

Сокращая приведённое выражение, получим:

$$
\begin{aligned}
& U_{\mathrm{ij}}=L s ?_{\mathrm{i}} \sqcup L s ?_{\mathrm{j}} \sqcup L s !_{\mathrm{j}}-\mathbf{L} s ?_{\mathrm{j}}=\mathbf{L s} ?_{\mathrm{i}} \sqcup \mathbf{L} s !_{\mathrm{j}}{ } \\
& \mathbf{U}_{\mathrm{ij}}=\mathbf{L s} ?_{\mathrm{i}} \sqcup \mathbf{L s} !_{\mathrm{j}} \text {; }
\end{aligned}
$$

Функционально система $\boldsymbol{S}_{\mathbf{i j}}$ преобразует входной поток $\mathbf{L} \boldsymbol{s} ?_{\mathbf{i}}$ системы $\boldsymbol{S}_{\mathbf{i}}$ в выходной поток $\mathbf{L} \boldsymbol{s}_{\mathbf{j}}$ системы $\boldsymbol{S}_{\mathbf{j}}$. Взаимодействие процессов систем $\boldsymbol{S}_{\mathbf{i}}$ и $\boldsymbol{S}_{\mathbf{j}}$ происходит вследствие того, что процесс $\boldsymbol{S}_{\mathbf{j}}$ использует результаты процесса $\boldsymbol{S}_{\mathbf{i}}$.

Таким образом, функция:

$$
\mathbf{F}_{\mathbf{i j}}=\mathbf{F}_{\mathbf{j}}\left(\mathbf{F}_{\mathbf{i}}\left(\mathbf{L} \boldsymbol{s} ?_{\mathbf{i}}\right)\right) \mathbf{L} \boldsymbol{s} !_{\mathbf{j}}=\mathbf{L} \boldsymbol{s} !_{\mathbf{j}} \sqcap \text { ЭимеетСоответствие. } \mathbf{L} \boldsymbol{s} ?_{\mathbf{i}} \text {. }
$$

Субстанциально система $\boldsymbol{S}_{\mathrm{ij}}$ имеет входной интерфейс $\mathbf{0}$ ? системы $\boldsymbol{S}_{\mathbf{i}}$ и выходной интерфейс $\mathbf{0} !_{\mathbf{j}}$ системы $\boldsymbol{S}_{\mathbf{j}}$. Кроме того, собственно объектные характеристики системы $\boldsymbol{S}_{\mathbf{i j}}$

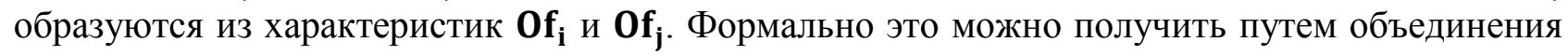
всех объектных характеристик системы $\boldsymbol{S}_{\mathbf{i}}$ и объектных характеристик системы $\boldsymbol{S}_{\mathbf{j}} \mathbf{c}$ последующим вычитанием из этого объединения результата пересечения объектных характеристик $\boldsymbol{S}_{\mathbf{i}}$ и $\mathbf{S}_{\mathbf{j}}$. Это позволит удалить из рассмотрения на уровне нового соединенного узлового объекта (системы $\boldsymbol{S}_{\mathrm{ij}}$ ) внутренние интерфейсные объектные характеристики, за счет которых происходит присоединение $\boldsymbol{S}_{\mathbf{i}}$ к $\boldsymbol{S}_{\mathbf{j}}$, по аналогии с потоковыми характеристиками (считая соответствующие друг другу характеристики равными).

Таким образом, объект

$$
\begin{aligned}
& \mathbf{O}_{\mathbf{i j}}=\left(O S ?_{\mathbf{i}} \sqcup O S !_{\mathbf{i}} \sqcup O S f_{i}\right) \sqcup\left(O S ?_{j} \sqcup O S !_{j} \sqcup O S f_{j}\right)-\left(O S ?_{i} \sqcup O S !_{i} \sqcup O S f_{i}\right) \sqcap \\
& \sqcap\left(O S ?_{j} \sqcup O S !_{j} \sqcup O S f_{j}\right)=O S ?_{i} \sqcup O S !_{j} \sqcup O S f_{i} \sqcup O S f_{j}=0 S ?_{i} \sqcup O S !_{j} \sqcup O S f_{i j}
\end{aligned}
$$


Тогда операция соединения систем выглядит следующим образом:

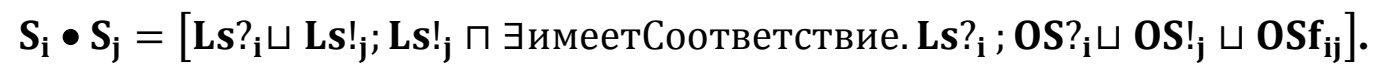

Операция объединения систем по входу.

Формально объединение систем по входу можно получить путем объединения потоков/связей системы $\boldsymbol{S}_{\mathbf{i}}$ и потоков/связей системы $\boldsymbol{S}_{\mathbf{j}}$. Данная операция осуществима при соблюдении следующих условий: $\mathbf{L s} ?_{\mathrm{i}}=\mathbf{L s} ?_{\mathrm{j}}, \operatorname{ran} \mathbf{F}_{\mathbf{S}_{\mathrm{i}}}=\boldsymbol{\operatorname { r a n }} \mathbf{F}_{\mathbf{S}_{\mathrm{j}}}, \mathbf{O S} ?_{\mathrm{i}}=\mathbf{O S} ?_{\mathbf{j}}$.

Таким образом, узел: $\mathbf{U}_{\mathbf{i j}}=\mathbf{L s} ?_{\mathbf{i}} \sqcup \mathbf{L s} !_{\mathbf{i}} \sqcup \mathbf{L s} ?_{\mathbf{j}} \sqcup \mathbf{L s} !_{\mathbf{j}}=\mathbf{L s} ?_{\mathbf{i}} \sqcup \mathbf{L s} !_{\mathbf{i}} \sqcup \mathbf{L s} !_{\mathbf{j}}$.

Функционально система $\boldsymbol{S}_{\mathbf{i j}}$ преобразует входной поток $\mathbf{L} \boldsymbol{s} ?_{\mathbf{i}}\left(\right.$ он же $\mathbf{L} \boldsymbol{s} ?_{\mathbf{j}}$ ) в выходные потоки $\mathbf{L} \boldsymbol{s} ?_{\mathbf{i}}$ и $\mathbf{L} \boldsymbol{s} ?_{\mathbf{j}}$ систем $\boldsymbol{S}_{\mathbf{i}}$ и $\boldsymbol{S}_{\mathbf{j}}$ путем параллельного выполнения процессов этих систем. Таким образом функция:

$$
\mathbf{F}_{\mathbf{i j}}=\mathbf{F}_{\mathbf{j}}\left(\mathbf{F}_{\mathbf{i}}\left(\mathbf{L} \boldsymbol{s} ?_{\mathbf{i}}\right)\right) \mathbf{L} \boldsymbol{s} !_{\mathbf{i}} \mathbf{L} \boldsymbol{s} !_{\mathbf{j}}=\left(\mathbf{L s} !_{\mathbf{j}} \sqcup \mathbf{L} \boldsymbol{s} !_{\mathbf{j}}\right) \sqcap \text { ヨимеетСоответствие. } \mathbf{L} \boldsymbol{s} ?_{\mathbf{i}} .
$$

Объектные характеристики системы образуются из характеристик $\mathbf{O S f}_{\mathbf{i}}$ и $\mathbf{O S f}_{\mathbf{j}}$ и получаются путем объединения всех объектных характеристик системы $\mathbf{S}_{\mathbf{i}}$ и объектных характеристик системы $\boldsymbol{S}_{\mathbf{j}}$.

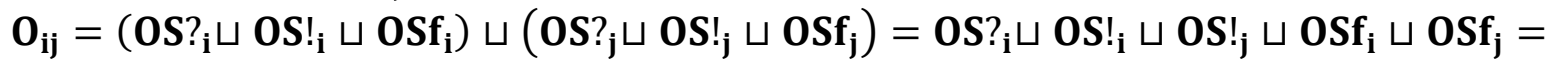

$$
\begin{aligned}
& =0 S ?_{\mathbf{i}} \sqcup 0 S !_{\mathbf{i}} \sqcup 0 S !_{\mathbf{j}} \sqcup \mathbf{O S f _ { i j }} \text {. }
\end{aligned}
$$

C учётом вышесказанного, операция объединения по входу может быть записана следующим образом:

$$
\begin{aligned}
& \mathbf{S}_{\mathbf{i}} \bullet \cap \mathbf{S}_{\mathbf{j}}=\left[\mathbf{L s} ?_{\mathbf{i}} \sqcup \mathbf{L s} !_{\mathbf{i}} \sqcup \mathbf{L s} !_{\mathbf{j}} ;\left(\mathbf{L s} !_{\mathbf{j}} \sqcup \mathbf{L s} !_{\mathbf{i}}\right) \sqcap \exists \text { имеетСоответствие. } \mathbf{L} \boldsymbol{s} ?_{\mathbf{i}} ; \mathbf{O S} ?_{\mathbf{i}} \sqcup \mathbf{O S} !_{\mathbf{i}} \sqcup \mathbf{O S} !_{\mathbf{j}} \sqcup\right. \\
& \left.\sqcup \mathbf{O S f}_{\mathbf{i j}}\right] \text {. }
\end{aligned}
$$

Операция объединения систем по выходу.

Операция выполняется при соблюдении следующих условий: $\mathbf{L s} !_{\mathbf{i}}=\mathbf{L s} !_{\mathbf{j}}, \boldsymbol{d o m} \mathbf{F}_{\mathbf{S}_{\mathbf{i}}}=$ $=\boldsymbol{d o m} \mathbf{F}_{\mathbf{S}_{\mathbf{j}}}, \mathbf{O S} !_{\mathbf{i}}=\mathbf{O S} !_{\mathbf{j}}$ По аналогии с операцией объединения систем по входу операция объединения систем по выходу может быть записана следующим образом:

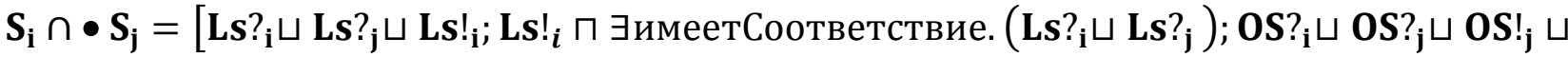

$$
\begin{aligned}
& \left.\sqcup \mathbf{O S f}_{\mathbf{i j}}\right] \text {. }
\end{aligned}
$$

\section{4. Пример использования нормативной системы при построении графоаналитических моделей}

Используя представленную выше нормативную систему, можно строить различные графоаналитические модели. Продемонстрируем возможности предлагаемой нормативной системы на примере простой модели в стандарте функционального моделирования IDEF0 (см. рис 2 и 3 ).

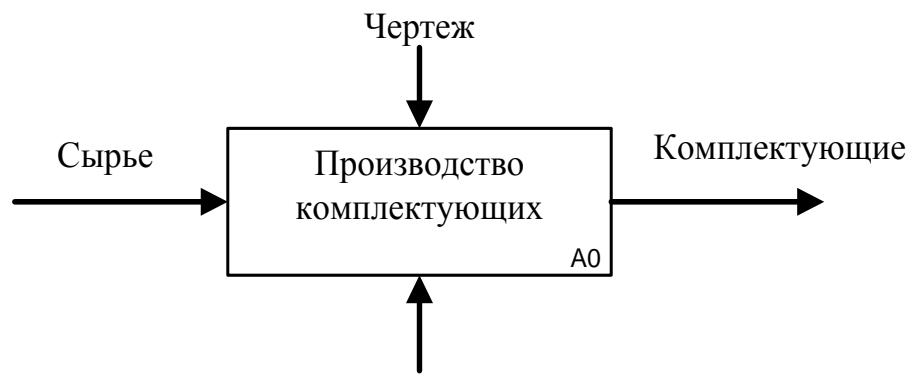

Оборудование

Рис. 2. Контекстная диаграмма производственного процесса Fig. 2. Context diagram of the production process 
Данную модель, уточняя результаты, представленные в работе [Маторин, 2018], можно представить с помощью нормативной системы системно-объектного моделирования (см. рис. 4 и 5). На рисунке 4 использованы следующие условные обозначения: VC - поток сырья; VК - поток результирующих комплектующих; СЧ - управляющая информация в виде чертежа. Подчеркнем, что в рамках системно-объектного подхода стрелка «механизм» (в данном случае «оборудование») не используется, так как системы, как конструкции «УзелФункция-Объект», сами по себе имеют объектные характеристики, которые здесь не показаны.

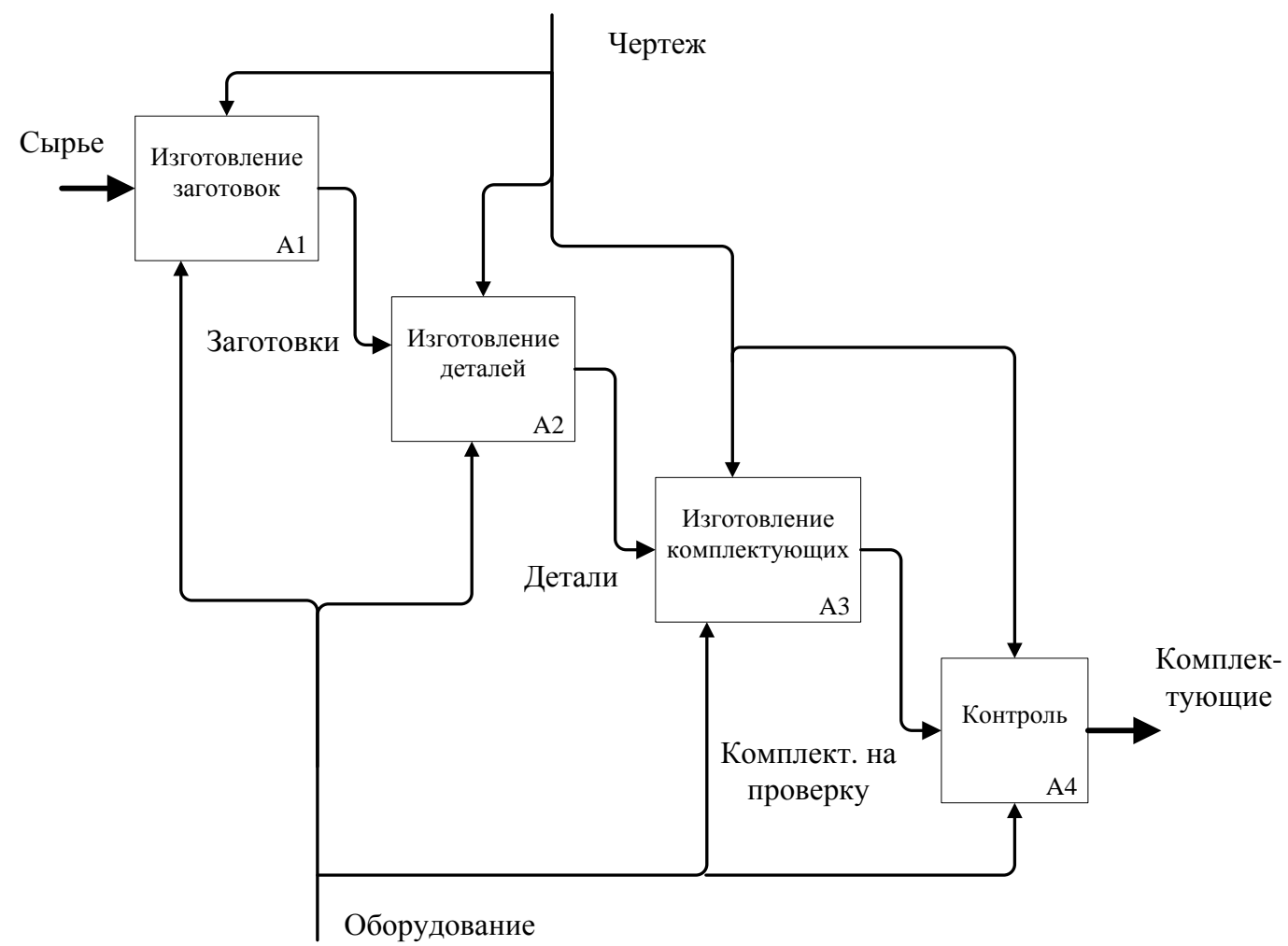

Рис. 3. Диаграмма декомпозиции производственного процесса

Fig. 3. The diagram of the decomposition of the production process

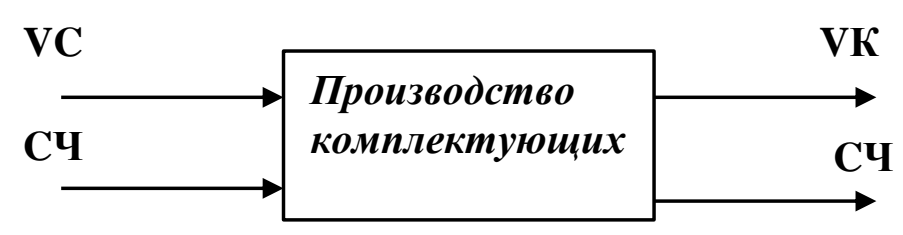

Рис. 4. Контекстная диаграмма производственного процесса как алфавитный элемент VC

Fig. 4. The context diagram of the production process as an alphabetical element of VC

Данную диаграмму можно представить в виде выражения (2) с пустой объектной составляющей:

$$
\begin{aligned}
& \text { ПроизводствоКомплектующих = [VC? ப CЧ?ப VК! ப СЧ!; } \\
& \text { (VК! ப СЧ!) П ЭобразуютКомплектующие. (VC? ப CЧ? ); ] }
\end{aligned}
$$

На рисунке 5 представлена графоаналитическая модель производственного процесса в терминах алфавитных УФО-элементов. Использованы следующие условные обозначения: VC, V3, VД, VКкон, VК - соответственно вещественные потоки сырья, заготовок, деталей, комплектующих на контроль и результирующих комплектующих; СЧ - управляющая информация в виде чертежа. 


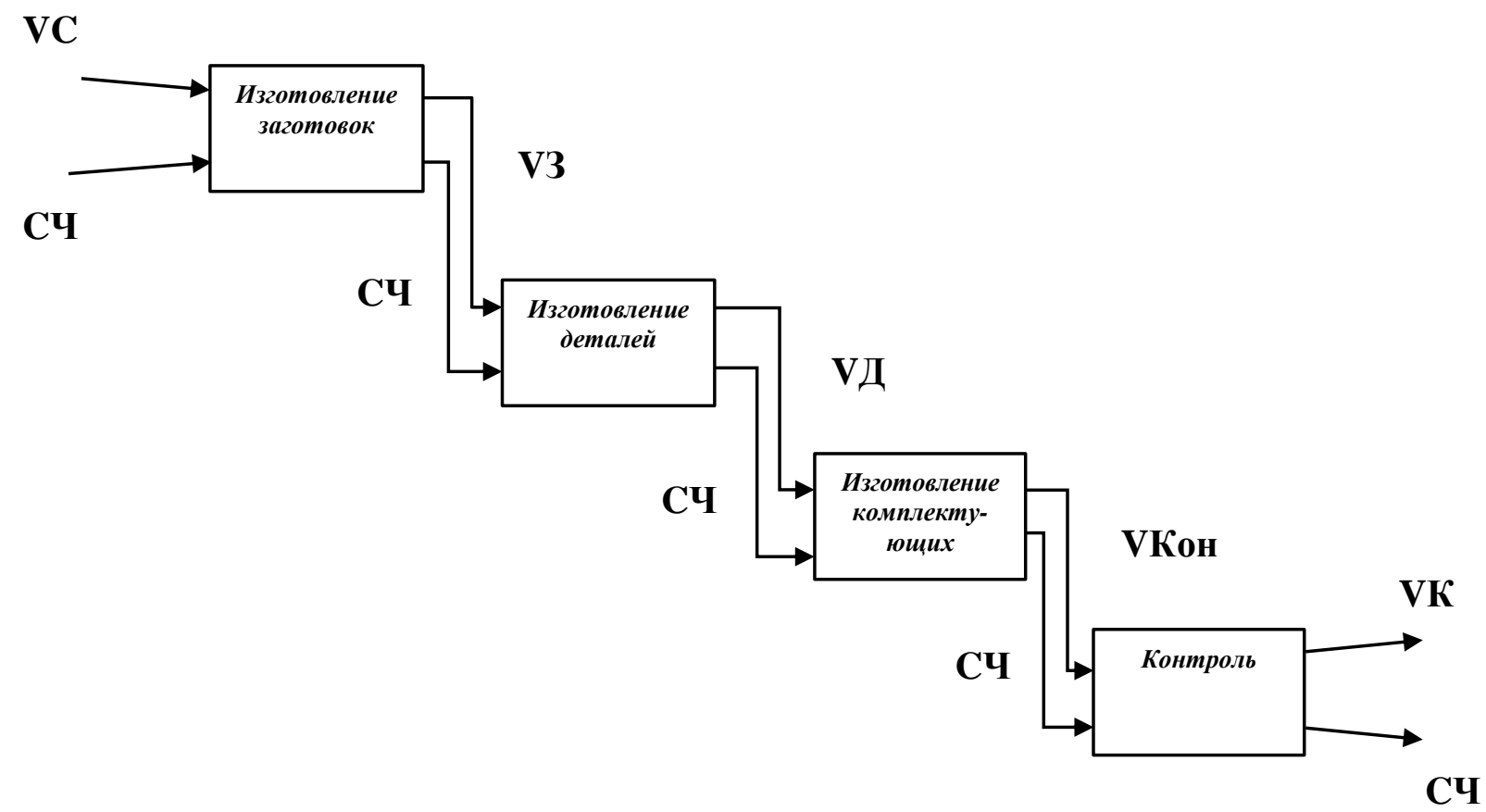

Рис. 5. Диаграмма декомпозиции производственного процесса как набор алфавитных элементов VC

Fig. 5. Diagram of the decomposition of the production process as a set of alphabetical elements VC

Представленные выше функциональные блоки можно описать с помощью ДЛ в виде выражения (2) с пустой объектной частью.

ИзготовлениеЗаготовок = [VC? ப CЧ? V3! ப СЧ!;

(V3! ப СЧ!) П ЭобразуютЗаготовки. (VC? ப СЧ? ); ]

ИзготовлениеДеталей = [V3? ப СЧ?ப VД! ப СЧ!;

(VД! ப СЧ!) П ЭобразуютДетали. (V3? ப CЧ?); ]

ИзготовлениеКомплектующих = [VД? ப CЧ? VКкон! ப СЧ!;

(VКкон! ப СЧ!) П ЭобразуютКомплектующие. (VД? ப СЧ?); ]

Контроль = [VКкон? ப СЧ? VК! ப СЧ!;

(VК! ப СЧ!) п ヨнаКонтроле. (VКкон? ப СЧ?); ]

Следовательно, типовой процесс производства комплектующих может быть представлен с помощью операции соединения алфавитных элементов типа VC (без учета правил баланса и реализации):

ПроизводствоКомплектующих = ИзготовлениеЗаготовок • ИзготовлениеДеталей •

• ИзготовлениеКомплектующих • Контроль.

\section{Заключение}

Предложен способ формирования адаптивного формально-семантического алфавита на основе категориальной иерархии классов связей/отношений, а также правила манипулирования им. Представленный алфавит и сформулированные правила представляют собой формально-семантическую нормативную систему системно-объектного анализа и моделирования. Такая нормативная система может использоваться для построения системнообъектных моделей, например, организационных систем. Это достигается путём декомпозиции таких систем на классы и объекты с помощью предложенного алфавита, что представляет собой этап объектно-ориентированного анализа и проектирования. 
Представленные отдельные виды функциональных элементов и связей для различных видов организационных систем обеспечивают имитацию понимания компьютером свойств классов и экземпляров, используемых для построения моделей. Таким образом, использование нормативной системы объективизирует результат построения моделей. Кроме того, предлагаемые алфавитные модельные элементы направляют процесс моделирования, уменьшая разнообразие возможных вариантов.

Для примера, представлена модель типового производственного процесса, полученная с помощью формально-семантической нормативной системы. Представленная нормативная система и ее формализация с помощью дескрипционной логики позволяют формально описывать графоаналитические модели сложных систем с помощью агрегации простых алфавитных элементов, что в перспективе позволяет решить задачу автоматизации системнообъектного графоаналитического моделирования.

\section{Список литературы}

1. Гильберт Д., Бернайс П. 1979. Основания математики. Том I. Логические исчисления и формализация арифметики. М.: Наука, 560.

2. Горский Д.П. 1990. О некотором способе введения понятий в «Капитале» Маркса. Сб. труд. междун. симпоз.: Исследования по логике научного познания. М.: Наука,. С.53-62.

3. Жихарев А.Г., Маторин С.И., Зайцева Н.О. 2015. Системно-объектный инструментарий для имитационного моделирования технологических процессов и транспортных потоков. Искусственный интеллект и принятие решений. № 4: 95-103.

4. Кондаков Н.И. 1975. Логический словарь-справочник. М.: Наука, 720.

5. Маслов С.Ю. 1986. Теория дедуктивных систем и ее применение. М.: «Радио и связь», 136.

6. Маторин С.И., Зимовец О.А., Щербинина Н.В., Сульженко Т.С. 2016. Концепция формализованной теории систем, основанной на подходе «Узел-Функция-Объект». Научные ведомости БелГУ. Серия Экономика. Информатика. 16 (237). 39: 159-166.

7. Маторин С.И., Жихарев А.Г., Игрунов К.К. 2018. Классификация систем как элементов «Узел-Функция-Объект». Научный результат. 3 (3): 15-27.

8. Мельников Г.П. 1978. Системология и языковые аспекты кибернетики. М.: Сов. радио, 368.

9. Михелев В.В., Маторин С.И. 2019. Формализация системно-объектного подхода с использованием дескрипционной логики. Научные ведомости БелГУ. Сер. Экономика. Информатика. 46 (2): 296-304.

10. Никаноров С.П. 1969. Системный анализ: этап развития методологии решения проблем в США. Вступ. статья в кн.: Оптнер С.Л. Системный анализ для решения деловых и промышленных проблем. Пер. с англ. М.: Советское радио, 7-45.

11. Петров Ю.А. 1977. Методологические вопросы анализа научного знания. М.: «Высш. школа», 224.

12. Степанов Ю.С. 1983. Семиотика. Под ред. Ю.С. Степанова. М.: Радуга, 640.

13. Теория систем и системный анализ: учебник. 2020. С.И. Маторин, А.Г. Жихарев, О.А. Зимовец и др.; под ред. С.И. Маторина. Москва; Берлин: Директмедиа Паблишинг, 509: Режим доступа: по подписке. URL: http://biblioclub.ru/index.php?page=book\&id=574641 (дата обращения: 12.06.2020).

14. Abadi Martin and Luca Cardelli 1996. A Theory of Objects. New York: Springer-Verlag, 397.

15. Baader F., Calvanese D., McGuinness L., Nardi D. Patel-Schneider P. F. 2003. The Description Logic Handbook: Theory, Implementation, and Applications. Cambridge University Press, 576.

16. Baader F., Sattler U. 1999. Expressive Number Restrictions in Description Logics. Journal of Logic and Computation. 9(3): 319-350.

17. Matorin S.I. 1998. Systems-Theoretic Investigation Of The Structure Of Categories. Automatic Document and Mathematical Linguistics. New York: Allerton Press, Inc. 31 (2): 4-9.

18. Matorin S.I. 1998. Modelling Intelligent Understanding Of The Language Of Business Communication. Automatic Document and Mathematical Linguistics. New York: Allerton Press, Inc. 31 (2): $47-58$.

19. Matorin S., Popov S., Matorin V. 2005. Organization Simulation Technology in The Light Of a New "Unit-Function-Object" Approach. Automatic Document and Mathematical Linguistics. New York: Allerton Press, Inc. 39 (1): 1-8. 
20. Schmidt-Schauss M., Smolka G. 1991. Attributive concept descriptions with complements. Artificial Intelligence. 48 (1): 1-26.

21. Tessaris S. 2001. Questions and answers: Reasoning and querying in Description Logic ( $\mathrm{PhD}$ Thesis). University of Manchester, 368.

\section{References}

1. Gil'bert D., Bernajs P. 1979. Osnovanija matematiki [Foundations of mathematics]. Tom I. Logicheskie ischislenija i formalizacija arifmetiki [Logical calculus and formalization of arithmetic.]. M.: Nauka, 560.

2. Gorskij D.P. 1990. O nekotorom sposobe vvedenija ponjatij v «Kapitale» Marksa [About some way of introducing concepts in Marx's "Capital"]. Sb. trud. mezhdun. simpoz.: Issledovanija po logike nauchnogo poznanija [Research on the logic of scientific knowledge]. M.: Nauka, 53-62.

3. Zhiharev A.G., Matorin S.I., Zajceva N.O. 2015. Sistemno-objektnyj instrumentarij dlja imitacionnogo modelirovanija tehnologicheskih processov i transportnyh potokov [System-object tools for simulation of technological processes and traffic flows]. Iskusstvennyj intellekt i prinjatie reshenij [Artificial Intelligence and Decision Making]. № 4: 95-103.

4. Kondakov N.I. 1975. Logicheskij slovar'-spravochnik [Logical dictionary-reference]. M.: Nauka, 720.

5. Maslov S.Ju. 1986. Teorija deduktivnyh sistem i ee primenenie [Theory of deductive systems and its application]. M.: «Radio i svjaz'», 136.

6. Matorin S.I., Zimovec O.A., Shherbinina N.V., Sul'zhenko T.S. 2016. Koncepcija formalizovannoj teorii sistem, osnovannoj na podhode «Uzel-Funkcija-Objekt» [The concept of a formalized theory of systems based on the approach "Node-Function-Object"]. Nauchnye vedomosti BelGU. Serija Jekonomika. Informatika [Scientific statements of BelSU. Series Economics. Informatics]. 16 (237). 39: 159-166.

7. Matorin S.I., Zhiharev A.G., Igrunov K.K. 2018. Klassifikacija sistem kak jelementov «UzelFunkcija-Objekt» [Classification of systems as elements "Unit-Function-Object"]. Nauchnyj rezul'tat [Scientific result]. 3 (3): 15-27.

8. Mel'nikov G.P. 1978. Sistemologija i jazykovye aspekty kibernetiki [Systemology and linguistic aspects of cybernetics]. M.: Sov. radio, 368.

9. Mihelev V.V., Matorin S.I. 2019. Formalizacija sistemno-objektnogo podhoda s ispol'zovaniem deskripcionnoj logiki [Formalization of a system-object approach using descriptive logic]. Nauchnye vedomosti BelGU. Ser. Jekonomika. Informatika [Scientific statements of BelSU. Series Economics. Informatics]. 46 (2): 296-304.

10. Nikanorov S.P. 1969. Sistemnyj analiz: jetap razvitija metodologii reshenija problem v SShA [System analysis: the stage of development of a problem solving methodology in the USA]. Vstup. stat'ja $\mathrm{v}$ kn.: Optner S.L. Sistemnyj analiz dlja reshenija delovyh i promyshlennyh problem [System analysis to solve business and industrial problems]. Per. s angl. M.: Sovetskoe radio, 7-45.

11. Petrov Ju.A. 1977. Metodologicheskie voprosy analiza nauchnogo znanija [Methodological issues of the analysis of scientific knowledge]. M.: «Vyssh. shkola», 224.

12. Stepanov Ju.S. 1983. Semiotika [Semiotics]. Pod red. Ju.S. Stepanova. M.: Raduga, 640.

13. Teorija sistem i sistemnyj analiz: uchebnik [Theory of systems and systems analysis: a textbook.] 2020. S.I. Matorin, A.G. Zhiharev, O.A. Zimovec i dr.; pod red. S.I. Matorina. Moskva; Berlin: Direktmedia Pablishing, 509: Access mode: by subscription. URL: http://biblioclub.ru/index.php?page=book\&id=574641 (date of the application: 12.06.2020).

14. Abadi Martin and Luca Cardelli 1996. A Theory of Objects. New York: Springer-Verlag, 397.

15. Baader F., Calvanese D., McGuinness L., Nardi D. Patel-Schneider P. F. 2003. The Description Logic Handbook: Theory, Implementation, and Applications. Cambridge University Press, 576.

16. Baader F., Sattler U. 1999. Expressive Number Restrictions in Description Logics. Journal of Logic and Computation. 9(3): 319-350.

17. Matorin S.I. 1998. Systems-Theoretic Investigation Of The Structure Of Categories. Automatic Document and Mathematical Linguistics. New York: Allerton Press, Inc. 31 (2): 4-9.

18. Matorin S.I. 1998. Modelling Intelligent Understanding Of The Language Of Business Communication. Automatic Document and Mathematical Linguistics. New York: Allerton Press, Inc. 31 (2): $47-58$. 
19. Matorin S., Popov S., Matorin V. 2005. Organization Simulation Technology in The Light Of a New "Unit-Function-Object" Approach. Automatic Document and Mathematical Linguistics. New York: Allerton Press, Inc. 39 (1): 1-8.

20. Schmidt-Schauss M., Smolka G. 1991. Attributive concept descriptions with complements. Artificial Intelligence. 48 (1): 1-26.

21. Tessaris S. 2001. Questions and answers: Reasoning and querying in Description Logic (PhD Thesis). University of Manchester, 368.

\section{ИНФОРМАЦИЯ ОБ АВТОРАХ}

Жихарев Александр Геннадиевич, кандидат технических наук, доцент кафедры информационно-телекоммуникационных систем и технологий Белгородского государственного национального исследовательского университета

Маторин Сергей Игоревич, доктор технических наук, профессор, заместитель генерального директора по науке и инновациям ЗАО «СофтКоннект», Белгород, Россия

Михелев Владимир Владимирович, аспирант кафедры прикладной информатики и информационных технологий Белгородского государственного национального исследовательского университета, Белгород, Россия

\section{INFORMATION ABOUT THE AUTHORS}

Alexander G. Zhikharev, Candidate of Technical Sciences, Associate Professor, Department of Information and Robotic Systems, Belgorod National Research University, Belgorod, Russia

Sergey I. Matorin, Doctor of Technical Sciences Professor, Deputy General Director on Science and Innovation CJSC "SoftConnect", Belgorod, Russia

Vladimir V. Mikhelev, Postgraduate Student, Department of Applied Informatics and Information Technologies, Belgorod National Research University, Belgorod, Russia 